\title{
Infinitely Many Nontrivial Solutions of Resonant Cooperative Elliptic Systems with Superlinear Terms
}

\author{
Guanwei Chen ${ }^{1}$ and Shiwang $\mathrm{Ma}^{2}$ \\ ${ }^{1}$ School of Mathematics and Statistics, Anyang Normal University, Anyang, Henan 455000, China \\ ${ }^{2}$ School of Mathematical Sciences and LPMC, Nankai University, Tianjin 300071, China \\ Correspondence should be addressed to Guanwei Chen; guanweic@163.com
}

Received 4 March 2014; Accepted 11 May 2014; Published 9 June 2014

Academic Editor: Adrian Petrusel

Copyright (c) 2014 G. Chen and S. Ma. This is an open access article distributed under the Creative Commons Attribution License, which permits unrestricted use, distribution, and reproduction in any medium, provided the original work is properly cited.

We study a class of resonant cooperative elliptic systems and replace the Ambrosetti-Rabinowitz superlinear condition with general superlinear conditions. We obtain ground state solutions and infinitely many nontrivial solutions of this system by a generalized Nehari manifold method developed recently by Szulkin and Weth.

\section{Introduction and Main Results}

We consider the following cooperative elliptic system:

$$
\begin{array}{cc}
-\Delta u=\xi u+f(x, u, v) & \text { in } \Omega, \\
-\Delta v=\zeta v+g(x, u, v) & \text { in } \Omega, \\
u=v=0 \quad \text { on } \partial \Omega, &
\end{array}
$$

where $\Omega$ is a bounded smooth domain in $\mathbb{R}^{N}$ and $\xi, \zeta \in \mathbb{R}$. The nonlinearities $(f, g)$ are the gradient of some function; that is, there exists a function $F \in C^{1}\left(\bar{\Omega} \times \mathbb{R}^{2}, \mathbb{R}\right)$ such that $\nabla F=(f, g)$. For system (1), we are interested in the resonant case; that is,

$$
\sigma\left(A^{*}\right) \cap \sigma(-\Delta) \neq \emptyset,
$$

where $A^{*}=\left(\begin{array}{ll}\xi & 0 \\ 0 & \zeta\end{array}\right), \sigma\left(A^{*}\right)=\{\xi, \zeta\}$ denotes the spectrum of the matrix $A^{*}$, and $\sigma(-\Delta)=\left\{\lambda_{k}: k=1,2, \ldots\right.$ and $0<\lambda_{1}<$ $\left.\lambda_{2}<\cdots\right\}$ denotes the eigenvalues of the Laplacian on $\Omega$ with zero boundary condition.

$$
\text { If } \xi=\zeta=\lambda_{k} \in \sigma(-\Delta), f(x, u, v)=\bar{f}(x, u), g(x, u, v)=
$$
$\bar{g}(x, v)$, and $\bar{f} \equiv \bar{g}$ on $\bar{\Omega} \times \mathbb{R}$, then (1) reduces to the following single elliptic equation:

$$
\begin{gathered}
-\Delta u=\lambda_{k} u+\bar{f}(x, u) \quad \text { in } \Omega, \\
u=0 \quad \text { on } \partial \Omega .
\end{gathered}
$$

The authors $[1,2]$ have considered the strongly resonant single elliptic equation (3) with odd nonlinearities and obtained a finite number of solutions. Li and Zou [3] investigated (3) by using the Morse theory.

The system (1) has been studied by many authors under asymptotically linear or sublinear assumptions on nonlinearities; see [4-10]. In [4], the variational structure was established and several existence results were obtained by minimax techniques under a condition which was called nonquadraticity at infinity. Ma [5] established the existence of infinitely many solutions for (1) with odd nonlinearities by the minimax techniques. Ma [6] and Zou et al. [9] established the existence and multiplicity of solutions for (1) via the computations of the critical groups and the Morse theory. By using a penalization technique and the Morse theory, Pomponio [7] established the existence and multiplicity of solutions of (1). However, very little is known about the existence of infinitely many solutions for resonant single elliptic equation and elliptic systems (both cooperative and noncooperative). Zou [8] considered (1) and, by using the methods used in [1], obtained infinitely many solutions under the oddness and boundedness assumptions on the nonlinearities. Zou [10] proved that (1) has infinitely many solutions under the oddness assumption and some growth assumptions near $U=0$. Recently, the author [11] also obtained the existence of ground state solutions for (1) in the nonresonant case (i.e., $\sigma\left(A^{*}\right) \cap \sigma(-\Delta)=\emptyset$ ) by a variant weak 
linking theorem. For related topics, including noncooperative elliptic systems, we refer the readers to [12-18] and references cited therein.

Let $|\cdot|$ and $(\cdot, \cdot)$ denote, respectively, the usual norm and the inner product in $\mathbb{R}^{2}$. Assume that

$\left(S_{0}\right)(\nabla F(x, U), Z)(U, Z) \geq 0$ for all $U, Z \in \mathbb{R}^{2}, \forall x \in \Omega$;

$\left(S_{1}\right)|\nabla F(x, U)| \leq a\left(1+|U|^{p-1}\right)$ for some $a>0$ and $p \epsilon$ $\left(2,2^{*}\right)$, where $2^{*}=2 N /(N-2)$ if $N \geq 3$ and $2^{*}:=+\infty$ if $N=1,2$

$\left(S_{2}\right)|\nabla F(x, U)|=o(|U|)$ as $|U| \rightarrow 0$ uniformly in $x$;

$\left(S_{3}\right) F(x, U) /|U|^{2} \rightarrow \infty$ as $|U| \rightarrow \infty$ uniformly in $x$;

$\left(S_{4}\right)(1 / 2)(\nabla F(x, U), U)>F(x, U)>0$ for all $U \in \mathbb{R}^{2} \backslash\{0\}$, $\forall x \in \Omega$;

$\left(S_{5}\right)|U|=|Z| \Rightarrow F(x, U)=F(x, Z)$ and $(\nabla F(x, U)$, $Z) \leq(\nabla F(x, U), U),|U|=|Z|$ and $U \neq Z \Rightarrow$ $(\nabla F(x, U), Z)<(\nabla F(x, U), U), \forall x \in \Omega$;

$\left(S_{6}\right)|U| \neq|Z|$ and $(U, Z) \neq 0 \Rightarrow(\nabla F(x, U), Z) \neq(\nabla F(x$, $Z), U), \forall x \in \Omega$.

In this paper, we obtain ground state solutions of (1), that is, nontrivial solutions corresponding to the least energy of the action functional of (1). Moreover, if $F(x, U)$ is even in $U$, we obtain infinitely many solutions of (1). Our main results based on a generalized Nehari manifold method developed in [2]. Now, our main results read as follows.

Theorem 1. If $\left(S_{0}\right)-\left(S_{6}\right)$ hold, then (1) admits a ground state solution.

Theorem 2. If $\left(S_{0}\right)-\left(S_{6}\right)$ and $F(x,-U)=F(x, U)$, for all $x \in \Omega$, hold, then (1) has infinitely many solutions $U_{k}$ such that $\left|U_{k}\right|_{\infty} \rightarrow \infty$.

Considering the following superquadratic condition there exists a constant $\mu>2$ such that

$$
0<\mu F(x, U) \leq(\nabla F(x, U), U), \quad x \in \Omega \backslash\{0\},
$$

which is now known as Ambrosetti-Rabinowitz superlinear condition. As we all know, our proofs will be more easier if we use the assumption (4). But we replace condition (4) with more general superquadratic conditions. As is shown in next examples, our assumptions $\left(S_{0}\right)-\left(S_{6}\right)$ are reasonable and there are cases in which Ambrosetti-Rabinowitz condition (4) is not satisfied.

Example 3. Let

$$
F(x, U)=f(x)|U|^{p},
$$

where $p>2$ and $f(x)>0$ is a continuous function. Clearly, $F$ satisfies $\left(S_{0}\right)-\left(S_{6}\right)$.

Example 4. Let

$$
F(x, U)=g(x)\left(|U|^{p}+(p-2)|U|^{p-\varepsilon} \sin ^{2}\left(\frac{|U|^{\varepsilon}}{\varepsilon}\right)\right),
$$

where $g(x)>0$ is a continuous function, $p>2,0<\varepsilon<p-2$ if $N=1,2$, and $0<\varepsilon<p+N-N p / 2$ if $N \geq 3$. Note that

$$
\begin{aligned}
\nabla F(x, U)=g(x) U[ & (p-2)(p-\varepsilon)|U|^{p-\varepsilon-2} \sin ^{2}\left(\frac{|U|^{\varepsilon}}{\varepsilon}\right) \\
& \left.+\left(p+(p-2) \sin \left(\frac{2|U|^{\varepsilon}}{\varepsilon}\right)\right)|U|^{p-2}\right] .
\end{aligned}
$$

It is not hard to check that $F$ satisfies $\left(S_{0}\right)-\left(S_{6}\right)$ but does not satisfy condition (4).

The rest of the present paper is organized as follows. In Section 2, we firstly establish the variational framework of (1), and then we give some preliminary lemmas, which are useful in the proofs of our main results. In Section 3, we give the detailed proofs of our main results.

\section{Variational Framework and Preliminaries}

Here and in what follows, we use $\|\cdot\|_{p}$ to denote the norm of $L^{p}(\Omega)$. Let

$$
E:=H_{0}^{1}(\Omega), \quad W:=H_{0}^{1}(\Omega) \times H_{0}^{1}(\Omega),
$$

where $H_{0}^{1}(\Omega)$ is the usual Sobolev space with the norm $\|\cdot\|_{E}$ generated by the inner product $\langle u, v\rangle_{E}=\int_{\Omega} \nabla u \nabla v d x$, for $u, v \in H_{0}^{1}(\Omega)$. For $U=\left(u_{1}, u_{2}\right)$ and $V=\left(v_{1}, v_{2}\right)$ in $W$, the induced inner product and norm on $W$ are given, respectively, by

$$
\begin{gathered}
\langle U, V\rangle_{W}=\left\langle u_{1}, v_{1}\right\rangle_{E}+\left\langle u_{2}, v_{2}\right\rangle_{E}, \\
\|U\|_{W}^{2}=\left\|u_{1}\right\|_{E}^{2}+\left\|u_{2}\right\|_{E}^{2} .
\end{gathered}
$$

Let $\vec{e}_{1}:=(1,0)$ and $\vec{e}_{2}:=(0,1)$; then $A^{*} \vec{e}_{1}=\xi \vec{e}_{1}$, $A^{*} \vec{e}_{2}=\zeta \vec{e}_{2}, \vec{e}_{1} \cdot \vec{e}_{2}=0$, and $\left|\vec{e}_{1}\right|=\left|\vec{e}_{2}\right|=1$. For any $\alpha \in \mathbb{R}$, let $H_{\alpha}^{+}, H_{\alpha}^{-}, H_{\alpha}^{0}$ be the subspaces of $H_{0}^{1}(\Omega)$, where the quadratic form $u \rightarrow\|u\|^{2}-\alpha\|u\|_{2}^{2}$ is positive definite, negative definite, and zero, respectively. Let

$$
\begin{gathered}
W^{0}:=H_{\xi}^{0} \times H_{\zeta}^{0}, \quad W^{+}:=H_{\xi}^{+} \times H_{\zeta}^{+}, \\
W^{-}:=H_{\xi}^{-} \times H_{\zeta}^{-} .
\end{gathered}
$$

Set

$$
A_{1}:=\mathbf{i d}-\xi(-\Delta)^{-1}, \quad A_{2}:=\mathbf{i d}-\zeta(-\Delta)^{-1},
$$

where id denotes the identity from $H_{0}^{1}(\Omega)$ to $H_{0}^{1}(\Omega)$. We introduce an operator

$$
\begin{array}{r}
A: W \longrightarrow W, \quad A=\left(A_{1}, A_{2}\right), \quad A U=\left(A_{1} u_{1}, A_{2} u_{2}\right) \\
\text { for any } U=\left(u_{1}, u_{2}\right) \in W .
\end{array}
$$

Then $A$ is a bounded self-adjoint operator from $W$ to $W$ and $\operatorname{ker} A=W^{0}$ with $\operatorname{dim} W^{0}<\infty$. The space $W$ splits 
as $W=W^{-} \oplus W^{0} \oplus W^{+}$, where $W^{-}$and $W^{+}$are invariant under $A,\left.A\right|_{W^{-}}$is negative, and $\left.A\right|_{W^{+}}$is positive definite. More precisely, there exists a positive constant $c_{0}$ such that

$$
\pm\left\langle A U^{ \pm}, U^{ \pm}\right\rangle_{W} \geq c_{0}\left\|U^{ \pm}\right\|_{W}^{2}, \quad \forall U^{ \pm} \in W^{ \pm} .
$$

Here and in what follows, for any $U \in W$, we always denote by $U^{0}, U^{+}$, and $U^{-}$the vectors in $W$ with $U=U^{0}+U^{-}+$ $U^{+}, U^{0} \in W^{0}$, and $U^{ \pm} \in W^{ \pm}$. Note that $\operatorname{dim} W^{0}$ and $\operatorname{dim} W^{-}$are finite. Furthermore, $\sigma\left(A^{*}\right) \cap \sigma(-\Delta) \neq \emptyset$ implies that $\operatorname{dim} W^{0} \neq 0$.

For problem (1), we consider the following functional:

$$
\begin{gathered}
\Phi(U)=\frac{1}{2}\langle A U, U\rangle_{W}-\int_{\Omega} \widetilde{F}(x, U) d x, \\
U=\left(u_{1}, u_{2}\right) \in W
\end{gathered}
$$

where $\widetilde{F}(x, s, t)=F\left(x, s \vec{e}_{1}+t \vec{e}_{2}\right)=F(x, s, t)$. We define an equivalent inner product $\langle\cdot, \cdot\rangle$ and the corresponding norm $\|\cdot\|$ on $W$ given, respectively, by

$$
\begin{gathered}
\langle U, V\rangle=\left\langle A U^{+}, V^{+}\right\rangle_{W}-\left\langle A U^{-}, V^{-}\right\rangle_{W}, \\
\|U\|=\langle U, U\rangle^{1 / 2},
\end{gathered}
$$

where $U=U^{-}+U^{0}+U^{+}, V=V^{-}+V^{0}+V^{+} \in W=$ $W^{-}+W^{0}+W^{+}$. Thus, $\Phi$ can be rewritten as

$$
\Phi(U)=\frac{1}{2}\left\|U^{+}\right\|^{2}-\frac{1}{2}\left\|U^{-}\right\|^{2}-\int_{\Omega} F(x, U) d x .
$$

Under our assumptions, $\Phi \in C^{1}(W, \mathbb{R})$ and the derivative is given by

$$
\Phi^{\prime}(U) V=\left\langle U^{+}, V^{+}\right\rangle-\left\langle U^{-}, V^{-}\right\rangle-\int_{\Omega}(\nabla F(x, U), V) d x,
$$

where $U=U^{-}+U^{0}+U^{+}, V=V^{-}+V^{0}+V^{+} \in W=$ $W^{-}+W^{0}+W^{+}$. By the discussion of [4], the (weak) solutions of system (1) are the critical points of the $C^{1}$ functional $\Phi$.

We let

$$
\begin{gathered}
\mathscr{M}:=\left\{U \in W \backslash\left(W^{-} \oplus W^{0}\right): \Phi^{\prime}(U) U=0,\right. \\
\left.\Phi^{\prime}(U) V=0, \forall V \in W^{-} \oplus W^{0}\right\} .
\end{gathered}
$$

By definition, it is easy to see that $\mathscr{M}$ contains all nontrivial critical points of $\Phi$. We define for $U \in W \backslash\left(W^{-} \oplus W^{0}\right)$ the subspace,

$$
W(U):=W^{-} \oplus W^{0} \oplus \mathbb{R} U=W^{-} \oplus W^{0} \oplus \mathbb{R} U^{+},
$$

and the convex subset,

$$
\widetilde{W}(U):=W^{-} \oplus W^{0} \oplus \mathbb{R}^{+} U=W^{-} \oplus W^{0} \oplus \mathbb{R}^{+} U^{+},
$$

of $W$, where $\mathbb{R}^{+}=[0, \infty)$.

We assume $\left(S_{0}\right)-\left(S_{6}\right)$ are satisfied from now on. Obviously, $\left(S_{1}\right)$ and $\left(S_{2}\right)$ imply that for each $\varepsilon>0$ there is $C_{\varepsilon}>0$ such that

$$
|\nabla F(x, U)| \leq \varepsilon|U|+C_{\varepsilon}|U|^{p-1}, \quad \forall U \in \mathbb{R}^{2} .
$$

Lemma 5. Let $Q:=s U+V \neq 0$, where $U, V \in \mathbb{R}^{2}, s \in \mathbb{R}$, and $s \geq-1$; then

$$
\begin{gathered}
\left(\nabla F(x, U), s\left(\frac{s}{2}+1\right) U+(s+1) V\right)+F(x, U) \\
-F(x, U+Q)<0, \quad \forall x \in \Omega .
\end{gathered}
$$

Proof. We fix $x \in \Omega$ and $U, V \in \mathbb{R}^{2}$. For $s \geq-1$, we let $Z=$ $Z(s):=(s+1) U+V$, so $Z=Q+U$. Let

$$
\begin{aligned}
h(s):= & \left(\nabla F(x, U), s\left(\frac{s}{2}+1\right) U+(s+1) V\right) \\
& +F(x, U)-F(x, Z) .
\end{aligned}
$$

We need to show that $h(s)<0$ whenever $Q=s U+V \neq 0$ (i.e., $U \neq Z$ ). We first consider the case $U=0$. Then $Z \neq 0$ and $h(s)=-F(x, Z)<0$ by $\left(S_{4}\right)$. We may therefore assume $U \neq 0$ from now on. It is not hard to check that $\left(S_{3}\right)$ and $\left(S_{4}\right)$ imply that

$$
\begin{aligned}
h(-1) & =-\frac{1}{2}(\nabla F(x, U), U)+F(x, U)-F(x, Z) \\
& <-F(x, Z) \leq 0, \quad \lim _{s \rightarrow \infty} h(s)=-\infty .
\end{aligned}
$$

Suppose that $h$ attains its maximum on $[-1, \infty)$ at some point $s$; then

$$
h^{\prime}(s)=(\nabla F(x, U), Z)-(\nabla F(x, Z), U)=0 .
$$

Case 1. If $(U, Z) \leq 0$, since $V=Z-(s+1) U$, it follows from $\left(S_{0}\right)$ and $\left(S_{4}\right)$ that

$$
\begin{aligned}
h(s)= & \left(\nabla F(x, U), s\left(\frac{s}{2}+1\right) U+(s+1)(Z-(s+1) U)\right) \\
& +F(x, U)-F(x, Z) \\
= & \left(-\frac{s^{2}}{2}-s-1\right)(\nabla F(x, U), U)+(s+1) \\
& \times(\nabla F(x, U), Z)+F(x, U)-F(x, Z) \\
< & -\frac{1}{2}(s+1)^{2}(\nabla F(x, U), U)+(s+1)(\nabla F(x, U), Z) \\
& -F(x, Z) \leq 0 .
\end{aligned}
$$

Case 2. If $(U, Z)>0$, it follows from $(25)$ and $\left(S_{6}\right)$ that $|U|=$ $|Z|$; it follows from the fact $U \neq Z,\left(S_{4}\right)$, and $\left(S_{5}\right)$ that

$$
\begin{aligned}
h(s)= & \left(\nabla F(x, U), s\left(\frac{s}{2}+1\right) U+(s+1)(Z-(s+1) U)\right) \\
& +F(x, U)-F(x, Z) \\
= & \left(-\frac{s^{2}}{2}-s-1\right)(\nabla F(x, U), U)+(s+1) \\
& \times(\nabla F(x, U), Z) \\
< & -\frac{s^{2}}{2}(\nabla F(x, U), U) \leq 0 .
\end{aligned}
$$

Therefore, $h(s)<0$ whenever $Q=s U+V \neq 0$. 
Lemma 6. Let $U \in \mathscr{M}$; then $\Phi(Q+U)<\Phi(U)$ for any $Q \in$ $H:=\left\{s U+V: s \geq-1, V \in W^{-} \oplus W^{0}\right\}, Q \neq 0$. Hence $U$ is the unique global maximum of $\left.\Phi\right|_{\widetilde{W}(U)}$.

Proof. Let $Q=s U+V \in H$ and $V=V^{-}+V^{0} \in W^{-} \oplus W^{0}$. Since $\Phi^{\prime}(U)=0$, we have

$$
\begin{aligned}
0= & \Phi^{\prime}(U)\left(s\left(\frac{s}{2}+1\right) U+(s+1) V\right) \\
= & s\left(\frac{s}{2}+1\right)\left\langle U^{+}, U^{+}\right\rangle-s\left(\frac{s}{2}+1\right)\left\langle U^{-}, U^{-}\right\rangle \\
& -(s+1)\left\langle U^{-}, V^{-}\right\rangle \\
& -\int_{\Omega}\left(\nabla F(x, U), s\left(\frac{s}{2}+1\right) U+(s+1) V\right) d x,
\end{aligned}
$$

which together with Lemma 5 and the facts $Q=s U+V$ and $\left\langle V^{-}, V^{-}\right\rangle \geq 0$ implies that

$$
\begin{aligned}
\Phi(Q+U)-\Phi(U) \\
=\frac{1}{2}\left[\left\langle(s+1) U^{+},(s+1) U^{+}\right\rangle-\left\langle U^{+}, U^{+}\right\rangle\right] \\
-\frac{1}{2}\left[\left\langle\left((s+1) U^{-}+V^{-}\right),(s+1) U^{-}+V^{-}\right\rangle\right. \\
\left.\quad-\left\langle U^{-}, U^{-}\right\rangle\right]+\int_{\Omega}[F(x, U)-F(x, Q+U)] d x \\
=s\left(\frac{s}{2}+1\right)\left\langle U^{+}, U^{+}\right\rangle-s\left(\frac{s}{2}+1\right)\left\langle U^{-}, U^{-}\right\rangle \\
-(s+1)\left\langle U^{-}, V^{-}\right\rangle-\frac{1}{2}\left\langle V^{-}, V^{-}\right\rangle \\
+\int_{\Omega}[F(x, U)-F(x, Q+U)] d x \\
=-\frac{1}{2}\left\langle V^{-}, V^{-}\right\rangle \\
+\int_{\Omega}\left[\left(\nabla F(x, U), s\left(\frac{s}{2}+1\right) U+(s+1) V\right)\right.
\end{aligned}
$$

The proof is finished.

In what follows, we let

$$
c:=\inf \{\Phi(U): U \in \mathscr{M}\} .
$$

Lemma 7. One has the following facts.

(a) There exists $\alpha>0$ such that $c \geq \inf _{S_{\alpha}} \Phi(U)>0$, where $S_{\alpha}:=\left\{U \in W^{+}:\|U\|=\alpha\right\}$.

(b) Consider $\left\|U^{+}\right\| \geq \max \left\{\left\|U^{-}\right\|, \sqrt{2 c}\right\}$ for every $U \in \mathscr{M}$.

Proof. (a) For $U \in W^{+}$, we have $\Phi(U)=(1 / 2)\|U\|^{2}-$ $\int_{\Omega} F(x, U) d x$ and $\int_{\Omega} F(x, U) d x=o\left(\|U\|^{2}\right)$ as $\|U\| \rightarrow 0$ by
(21) and $\left(S_{4}\right)$; hence the second inequality follows if $\alpha>0$ is sufficiently small. Since for every $U \in \mathscr{M}$ there is $s>0$ such that $s U^{+} \in \widetilde{W}(U) \cap S_{\alpha}$, the first inequality is a consequence of Lemma 6.

(b) For $U \in \mathscr{M}$, we have

$$
\begin{aligned}
c & \leq \frac{1}{2}\left(\left\|U^{+}\right\|^{2}-\left\|U^{-}\right\|^{2}\right)-\int_{\Omega} F(x, U) d x \\
& \leq \frac{1}{2}\left(\left\|U^{+}\right\|^{2}-\left\|U^{-}\right\|^{2}\right) ;
\end{aligned}
$$

thus $\left\|U^{+}\right\| \geq \max \left\{\left\|U^{-}\right\|, \sqrt{2 c}\right\}$. The proof is finished.

Lemma 8. If $D \subset W^{+} \backslash\{0\}$ is a compact subset, then there exists $R>0$ such that $\Phi \leq 0$ on $W(U) \backslash B_{R}(0)$ for every $U \in D$.

Proof. Without loss of generality, we may assume that $\|U\|=$ 1 for every $U \in D$. Suppose by contradiction that there exist $U_{n} \in D$ and $Q_{n} \in W\left(U_{n}\right)(n \in \mathbb{N})$ such that $\Phi\left(Q_{n}\right) \geq 0$ for all $n$ and $\left\|Q_{n}\right\| \rightarrow \infty$ as $n \rightarrow \infty$. Passing to a subsequence, we assume $U_{n} \rightarrow U \in W^{+},\|U\|=1$. Let $V_{n}=Q_{n} /\left\|Q_{n}\right\|=$ $s_{n} U_{n}+V_{n}^{-}+V_{n}^{0}$; then

$$
0 \leq \frac{\Phi\left(Q_{n}\right)}{\left\|Q_{n}\right\|^{2}}=\frac{1}{2}\left(s_{n}^{2}-\left\|V_{n}^{-}\right\|^{2}\right)-\int_{\Omega} \frac{F\left(x, Q_{n}\right)}{\left|Q_{n}\right|^{2}}\left|V_{n}\right|^{2} d x .
$$

Thus $\left\|V_{n}^{-}\right\|^{2} \leq s_{n}^{2}=1-\left\|V_{n}^{0}\right\|^{2}-\left\|V_{n}^{-}\right\|^{2}$.

Therefore, $s_{n} \rightarrow s \geq 0$ after passing to a subsequence. We may assume that $V_{n}(x) \rightarrow V(x)$ in $W$ and $V_{n}(x) \rightarrow V(x)$ a.e. in $\Omega$ after passing to a subsequence. If $s>0$, then $V=$ $s U+V^{-}+V^{0} \neq 0$. Hence $\left|Q_{n}\right|=\left|V_{n}\right|\left\|Q_{n}\right\| \rightarrow \infty$ as $n \rightarrow \infty$; it follows from $\left(S_{3}\right)$ and Fatou's lemma that

$$
\int_{\Omega} \frac{F\left(x, Q_{n}\right)}{\left|Q_{n}\right|^{2}}\left|V_{n}\right|^{2} d x \longrightarrow \infty \quad \text { as } n \longrightarrow \infty,
$$

which contradicts with (32). If $s=0$, then it follows from $\left\|V_{n}^{-}\right\|^{2} \leq s_{n}^{2}=1-\left\|V_{n}^{0}\right\|^{2}-\left\|V_{n}^{-}\right\|^{2}$ that $V_{n}^{-} \rightarrow 0$ and $V_{n}^{0} \rightarrow$ $V^{0} \neq 0$. Thus $V \neq 0$. Therefore, (33) still holds. We also get a contradiction. The proof is finished.

Lemma 9. For each $U \notin W^{0} \oplus W^{-}$, the set $\mathscr{M} \cap \widetilde{W}(U)$ consists of precisely one point $\widetilde{m}(U)$ which is the unique global maximum of $\left.\Phi\right|_{\widetilde{W}(U)}$.

Proof. By Lemma 6, it suffices to show that $\mathscr{M} \cap \widetilde{W}(U) \neq \emptyset$. Since $\widetilde{W}(U)=\widetilde{W}\left(U^{+}\right)$, we may assume that $U \in W^{+},\|U\|=$ 1. By Lemma 8, there exists $R>0$ such that $\Phi \leq 0$ on $W(U) \backslash$ $B_{R}(0)$. By Lemma $7(\mathrm{a}), \Phi(t U)>0$ for small $t>0$, and since $\Phi \leq 0$ on $\widetilde{W}(U) \backslash B_{R}(0), 0<\sup _{\widetilde{W}(U)} \Phi<\infty$. It is easy to see that $\Phi$ is weakly upper semicontinuous on $\widetilde{W}(U)$; therefore, $\Phi\left(U_{0}\right)=\sup _{\widetilde{W}(U)} \Phi$ for some $U_{0} \in \widetilde{W}(U) \backslash\{0\}$. This $U_{0}$ is a critical point of $\left.\Phi\right|_{W(U)}$, so $\Phi^{\prime}\left(U_{0}\right) U_{0}=\Phi^{\prime}\left(U_{0}\right) V=0$ for all $V \in W(U)$. Consequently, $U_{0} \in \mathscr{M} \cap \widetilde{W}(U)$, as required.

Lemma 10. $\Phi$ is coercive on $\mathscr{M}$; that is, $\Phi(U) \rightarrow \infty$ as $\|U\| \rightarrow \infty, U \in \mathscr{M}$. 
Proof. Arguing by contradiction, suppose that there exists a sequence $\left\{U_{n}\right\} \subset \mathscr{M}$ such that $\left\|U_{n}\right\| \rightarrow \infty$ and $\Phi\left(U_{n}\right) \leq d$ for some $d \in[c, \infty)$. Let $V_{n}=U_{n} /\left\|U_{n}\right\|$. Then $V_{n}(x) \rightarrow V(x)$ in $W$ and $V_{n}(x) \rightarrow V(x)$ a.e. in $\Omega$ after passing to a subsequence. By Lemma $7(\mathrm{~b}),\left\|V_{n}^{+}\right\|^{2} \geq 2 c$. By Sobolev compact embedding theorem, we get

$$
V_{n}^{+} \longrightarrow V^{+} \quad \text { in } L^{r}(\Omega) \times L^{r}(\Omega), 1 \leq r<2^{*} .
$$

If $V^{+}=0$, then it follows from (21), (34), and $\left(S_{4}\right)$ that $\int_{\Omega} F\left(x, s V_{n}^{+}\right) d x \rightarrow 0$ as $n \rightarrow \infty$ for every $s \in \mathbb{R}$. Since $s V_{n}^{+} \in \widetilde{W}\left(U_{n}\right)$ for $s \geq 0$, Lemma 6 implies that

$$
\begin{aligned}
d \geq & \Phi\left(U_{n}\right) \geq \Phi\left(s V_{n}^{+}\right) \\
= & \frac{s^{2}}{2}\left\|V_{n}^{+}\right\|^{2}-\int_{\Omega} F\left(x, s V_{n}^{+}\right) d x \geq c s^{2} \\
& -\int_{\Omega} F\left(x, s V_{n}^{+}\right) d x \longrightarrow c s^{2} \text { as } n \longrightarrow \infty .
\end{aligned}
$$

This yields a contradiction if $s>\sqrt{d / c}$. Hence $V^{+} \neq 0$, which implies that $V \neq 0$. Therefore, $\left|U_{n}\right|=\left|V_{n}\right| \mid U_{n} \| \rightarrow$ $\infty$; it follows again from $\left(S_{3}\right)$ and Fatou's lemma that $\int_{\Omega}\left(F\left(x, U_{n}\right) /\left|U_{n}\right|^{2}\right)\left|V_{n}\right|^{2} d x \rightarrow \infty$; therefore,

$$
\begin{aligned}
0 \leq & \frac{\Phi\left(U_{n}\right)}{\left\|U_{n}\right\|^{2}}=\frac{1}{2}\left(\left\|V_{n}^{+}\right\|^{2}-\left\|V_{n}^{-}\right\|^{2}\right) \\
& -\int_{\Omega} \frac{F\left(x, U_{n}\right)}{\left|U_{n}\right|^{2}}\left|V_{n}\right|^{2} d x \longrightarrow-\infty,
\end{aligned}
$$

as $n \rightarrow \infty$, a contradiction. The proof is finished.

Lemma 11. The map $W^{+} \backslash\{0\} \rightarrow \mathscr{M}, U \rightarrow \widetilde{m}(U)$ (see Lemma 9) is continuous.

Proof. Let $U \in W^{+} \backslash\{0\}$. By a standard argument, the continuity of $\widetilde{m}$ in $U$ is reduced to the following assertion:

$$
\text { If } U_{n} \longrightarrow U \text { for }\left\{U_{n}\right\} \subset W^{+} \backslash\{0\},
$$

then $\widetilde{m}\left(U_{n}\right) \longrightarrow \widetilde{m}(U)$ for a subsequence.

To prove the above assertion, we let $\left\{U_{n}\right\} \subset W^{+} \backslash\{0\}$ be a sequence with $U_{n} \rightarrow U$. Without loss of generality, we may assume that $\left\|U_{n}\right\|=\|U\|=1$ for all $n$, so that

$$
\widetilde{m}\left(U_{n}\right)=\left\|\widetilde{m}\left(U_{n}\right)^{+}\right\| U_{n}+\widetilde{m}\left(U_{n}\right)^{-}+\widetilde{m}\left(U_{n}\right)^{0} .
$$

By Lemma 8, there exists $R>0$ such that

$$
\begin{aligned}
\Phi\left(\widetilde{m}\left(U_{n}\right)\right) & =\sup _{W\left(U_{n}\right)} \Phi \leq \sup _{B_{R}(0)} \Phi \leq \sup _{U \in B_{R}(0)}\left\|U^{+}\right\|^{2} \\
& =R^{2} \quad \text { for every } n .
\end{aligned}
$$

Hence $\left\{\widetilde{m}\left(U_{n}\right)\right\}$ is bounded by Lemma 10 . Note that $\operatorname{dim} W^{0}$ and $\operatorname{dim} W^{-}$are finite. Passing to a subsequence, we may assume that

$$
\begin{aligned}
t_{n}:=\left\|\widetilde{m}\left(U_{n}\right)^{+}\right\| & \longrightarrow t, \quad \tilde{m}\left(U_{n}\right)^{-} \longrightarrow U_{1}^{-}, \\
\widetilde{m}\left(U_{n}\right)^{0} & \longrightarrow U_{1}^{0} \quad \text { in } W \text { as } n \longrightarrow \infty,
\end{aligned}
$$

where $t \geq \sqrt{2 c}>0$ by Lemma $7(\mathrm{~b})$. Therefore, we have

$$
\widetilde{m}\left(U_{n}\right) \longrightarrow t U+U_{1}^{-}+U_{1}^{0} \quad \text { in } W \text { as } n \longrightarrow \infty
$$

Note that $\widetilde{m}(U)=\left\|\widetilde{m}(U)^{+}\right\| U+\widetilde{m}(U)^{-}+\widetilde{m}(U)^{0}$. By Lemma 9, we have

$$
\begin{aligned}
\Phi\left(\widetilde{m}\left(U_{n}\right)\right) & \geq \Phi\left(\left\|\widetilde{m}(U)^{+}\right\| U_{n}+\widetilde{m}(U)^{-}+\widetilde{m}(U)^{0}\right) \\
& \longrightarrow \Phi\left(\left\|\widetilde{m}(U)^{+}\right\| U+\widetilde{m}(U)^{-}+\widetilde{m}(U)^{0}\right) \\
& =\Phi(\widetilde{m}(U)) \quad \text { as } n \longrightarrow \infty,
\end{aligned}
$$

which together with (40), (41), and Fatou's lemma implies that

$$
\begin{aligned}
\Phi & (\widetilde{m}(U)) \\
& \leq \lim _{n \rightarrow \infty} \Phi\left(\widetilde{m}\left(U_{n}\right)\right) \\
& =\lim _{n \rightarrow \infty}\left(\frac{1}{2} t_{n}^{2}-\frac{1}{2}\left\|\widetilde{m}\left(U_{n}\right)^{-}\right\|^{2}-\int_{\Omega} F\left(x, \widetilde{m}\left(U_{n}\right)\right) d x\right) \\
& \leq \frac{1}{2} t^{2}-\frac{1}{2}\left\|U_{1}^{-}\right\|^{2}-\int_{\Omega} F\left(x, t U+U_{1}^{-}+U_{1}^{0}\right) d x \\
& =\Phi\left(t U+U_{1}^{-}+U_{1}^{0}\right) .
\end{aligned}
$$

On the other hand, Lemma 9 implies that $\Phi\left(t U+U_{1}^{-}+U_{1}^{0}\right) \leq$ $\Phi(\widetilde{m}(U))$. Therefore, Lemma 9 implies that $\widetilde{m}(U)=t U+U_{1}^{-}+$ $U_{1}^{0}$. It follows from (41) that $\widetilde{m}\left(U_{n}\right) \rightarrow \widetilde{m}(U)$. The proof is finished.

We now consider the functional

$$
\widetilde{\Psi}: W^{+} \backslash\{0\} \longrightarrow \mathbb{R}, \quad \widetilde{\Psi}(U):=\Phi(\widetilde{m}(U)),
$$

which is continuous by Lemma 11 .

Lemma 12. Consider $\widetilde{\Psi} \in C^{1}\left(W^{+} \backslash\{0\}, \mathbb{R}\right)$ and $\widetilde{\Psi}^{\prime}(Q) Z=$ $\left(\left\|\widetilde{m}(Q)^{+}\right\| /\|Q\|\right) \Phi^{\prime}(\widetilde{m}(Q)) Z$ for $Q, Z \in W^{+}, Q \neq 0$.

Proof. We put $U=\widetilde{m}(Q) \in \mathscr{M}$, so we have $U=U^{-}+U^{0}+$ $\left(\left\|U^{+}\right\| /\|Q\|\right) Q$. Let $Z \in W^{+}$. Choose $\delta>0$ such that $Q_{t}:=$ $Q+t Z \in W^{+} \backslash\{0\}$ for $|t|<\delta$ and let $U_{t}=\widetilde{m}\left(Q_{t}\right) \in \mathscr{M}$. We may write $U_{t}=U_{t}^{-}+U_{t}^{0}+s_{t} Q_{t}$ with $s_{t}>0$. Then $s_{0}=\left(\left\|U^{+}\right\| /\|Q\|\right)$, and the function $(-\delta, \delta) \rightarrow \mathbb{R}, t \rightarrow s_{t}$, is continuous by Lemma 11. By Lemma 9 and the mean value theorem, we have

$$
\begin{aligned}
& \widetilde{\Psi}\left(Q_{t}\right)-\widetilde{\Psi}(Q) \\
& \quad=\Phi\left(U_{t}\right)-\Phi(U) \\
& \quad=\Phi\left(U_{t}^{-}+U_{t}^{0}+s_{t} Q_{t}\right)-\Phi\left(U^{-}+U^{0}+s_{0} Q\right) \\
& \quad \leq \Phi\left(U_{t}^{-}+U_{t}^{0}+s_{t} Q_{t}\right)-\Phi\left(U_{t}^{-}+U_{t}^{0}+s_{t} Q\right) \\
& \quad=s_{t}\left(Q_{t}-Q\right) \Phi^{\prime}\left(U_{t}^{-}+U_{t}^{0}+s_{t}\left(Q+\theta_{t}\left(Q_{t}-Q\right)\right)\right) \\
& \quad=s_{0} t \Phi^{\prime}(U) Z+o(t) \quad \text { as } t \longrightarrow 0,
\end{aligned}
$$


with some $\theta_{t} \in(0,1)$. By a similar reasoning, we also have

$$
\begin{aligned}
& \widetilde{\Psi}\left(Q_{t}\right)-\widetilde{\Psi}(Q) \\
& \quad=\Phi\left(U_{t}^{-}+U_{t}^{0}+s_{t} Q_{t}\right)-\Phi\left(U^{-}+U^{0}+s_{0} Q\right) \\
& \quad \geq \Phi\left(U^{-}+U^{0}+s_{0} Q_{t}\right)-\Phi\left(U^{-}+U^{0}+s_{0} Q\right) \\
& \quad=s_{0}\left(Q_{t}-Q\right) \Phi^{\prime}\left(U^{-}+U^{0}+s_{0}\left(Q+\eta_{t}\left(Q_{t}-Q\right)\right)\right) \\
& \quad=s_{0} t \Phi^{\prime}(U) Z+o(t) \quad \text { as } t \longrightarrow 0,
\end{aligned}
$$

with some $\eta_{t} \in(0,1)$. Combining (45) and (46), we conclude that the directional derivative $\partial_{Z} \widetilde{\Psi}(Q)$ exists and is given by

$$
\begin{aligned}
\partial_{Z} \widetilde{\Psi}(Q) & =\lim _{t \rightarrow 0} \frac{\widetilde{\Psi}\left(Q_{t}\right)-\widetilde{\Psi}(Q)}{t}=s_{0} \Phi^{\prime}(U) Z \\
& =\frac{\left\|\widetilde{m}(Q)^{+}\right\|}{\|Q\|} \Phi^{\prime}(\widetilde{m}(Q)) Z .
\end{aligned}
$$

Hence $\partial_{Z} \widetilde{\Psi}(Q)$ is linear (and continuous) in $Z$ and depends continuously on $Q$. So the assertion follows by $[19$, Proposition 1.3].

Next we consider the unit sphere $S^{+}:=\left\{Q \in W^{+}:\|Q\|=\right.$ $1\}$ in $W^{+}$. We note that the restriction of the map $\widetilde{m}$ to $S^{+}$is a homeomorphism with inverse given by

$$
\breve{m}: \mathscr{M} \longrightarrow S^{+}, \quad \breve{m}(U)=\frac{U^{+}}{\left\|U^{+}\right\|} .
$$

We also consider the restriction $\Psi: S^{+} \rightarrow \mathbb{R}$ of $\widetilde{\Psi}$ to $S^{+}$.

Lemma 13. One has the following facts:

(a) $\Psi \in C^{1}\left(S^{+}\right)$and $\Psi^{\prime}(Q) Z=\left\|\widetilde{m}(Q)^{+}\right\| \Phi^{\prime}(\widetilde{m}(Q)) Z$ for $Z \in T_{Q} S^{+}=\left\{V \in W^{+}:\langle Q, V\rangle=0\right\}$,

(b) $\left\{Q_{n}\right\}$ is a Palais-Smale sequence of $\Psi$ if and only if $\left\{\tilde{m}\left(Q_{n}\right)\right\}$ is a Palais-Smale sequence of $\Phi$,

(c) one has $\inf _{S^{+}} \Psi=c$. Moreover, $U \in S^{+}$is a critical point of $\Psi$ if and only if $\widetilde{m}(U) \in M$ is a critical point of $\Phi$, and the corresponding critical values coincide.

Proof. (a) is a direct consequence of Lemma 12.

To prove (b), let $\left\{Q_{n}\right\}$ be a sequence such that $C:=$ $\sup _{n} \Psi\left(Q_{n}\right)=\sup _{n} \Phi\left(\widetilde{m}\left(Q_{n}\right)\right)<\infty$, and let $U_{n}=\widetilde{m}\left(Q_{n}\right) \in \mathscr{M}$. Since for every $n$ we have an orthogonal splitting

$$
\begin{aligned}
W & =W\left(Q_{n}\right) \oplus T_{Q_{n}} S^{+} \\
& \left.=W\left(U_{n}\right) \oplus T_{Q_{n}} S^{+} \text {(with respect to }\langle\cdot, \cdot\rangle\right)
\end{aligned}
$$

and $\Phi^{\prime}\left(U_{n}\right) V=0$ for all $V \in W\left(U_{n}\right)$, we find that $\nabla \Phi\left(U_{n}\right) \in$ $T_{Q_{n}} S^{+}$and using (a),

$$
\begin{aligned}
\left\|\Psi^{\prime}\left(Q_{n}\right)\right\| & =\sup _{Z \in T_{\mathrm{Q}_{n}} S^{+},\|Z\|=1} \Psi^{\prime}\left(Q_{n}\right) Z \\
& =\sup _{Z \in T_{\mathrm{Q}_{n}} S^{+},\|Z\|=1}\left\|U_{n}^{+}\right\| \Phi^{\prime}\left(U_{n}\right) Z=\left\|U_{n}^{+}\right\|\left\|\Phi^{\prime}\left(U_{n}\right)\right\| .
\end{aligned}
$$

By Lemma 7(b) and Lemma 10, we have $\sqrt{2 c} \leq\left\|U_{n}^{+}\right\| \leq$ $\sup _{n}\left\|U_{n}^{+}\right\|<\infty$. Hence $\left\{Q_{n}\right\}$ is a Palais-Smale sequence for $\Psi$ if and only if $\left\{U_{n}\right\}$ is a Palais-Smale sequence for $\Phi$.

In (c) the proof is similar as in (b) but easier.

\section{Proofs of Main Results}

We are now in a position to prove our main results.

Proof of Theorem 1. From Lemma 7(a) we know that $c>0$. Moreover, if $U_{0} \in \mathscr{M}$ satisfies $\Phi\left(U_{0}\right)=c$, then $\breve{m}\left(U_{0}\right) \in S^{+}$is a minimizer of $\Psi$ and therefore a critical point of $\Psi$, so that $U_{0}$ is a critical point of $\Phi$ by Lemma 13. It remains to show that there exists a minimizer $U \in \mathscr{M}$ of $\left.\Phi\right|_{\mathscr{M}}$. By Ekeland's variational principle [19], there exists a sequence $\left\{Q_{n}\right\} \subset S^{+}$ with $\Psi\left(Q_{n}\right) \rightarrow c$ and $\Psi^{\prime}\left(Q_{n}\right) \rightarrow 0$ as $n \rightarrow \infty$. Put $U_{n}=$ $\widetilde{m}\left(Q_{n}\right) \in \mathscr{M}$ for $n \in \mathbb{N}$. Then $\Phi\left(U_{n}\right) \rightarrow c$ and $\Phi^{\prime}\left(U_{n}\right) \rightarrow 0$ as $n \rightarrow \infty$ by Lemma 13(b). By Lemma 10, $\left\{U_{n}\right\}$ is bounded and hence $U_{n} \rightarrow U$ in $W$ after passing to a subsequence. As a result of the Sobolev compact embedding theorem, we get

$$
U_{n} \longrightarrow U \text { in } L^{r}(\Omega) \times L^{r}(\Omega), 1 \leq r<2^{*} .
$$

If $U=0$, then it follows from (21), (51), and Hölder's inequality that

$$
\int_{\Omega}\left(\nabla F\left(x, U_{n}\right), U_{n}^{+}\right) d x \longrightarrow 0 \quad \text { as } n \longrightarrow \infty .
$$

Note that $\Phi^{\prime}\left(U_{n}\right) \rightarrow 0$ as $n \rightarrow \infty$ implies that

$$
\Phi^{\prime}\left(U_{n}\right) U_{n}^{+}=\left\|U_{n}^{+}\right\|^{2}-\int_{\Omega}\left(\nabla F\left(x, U_{n}\right), U_{n}^{+}\right) d x \longrightarrow 0
$$

$$
\text { as } n \longrightarrow \infty \text {. }
$$

Therefore, we have $\left\|U_{n}^{+}\right\| \rightarrow 0$ as $n \rightarrow \infty$, which is contrary to Lemma $7\left(\right.$ b). So $U \neq 0$ and $\Phi^{\prime}(U)=0$.

For any $U \in \mathscr{M}$, assumption $\left(S_{4}\right)$ implies that

$$
\begin{aligned}
c & =\Phi(U)-\frac{1}{2} \Phi^{\prime}(U) U \\
& =\int_{\Omega}\left(\frac{1}{2}(\nabla F(x, U), U)-F(x, U)\right) d x>0 \quad \text { if } U \neq 0 .
\end{aligned}
$$

Therefore, $c \geq 0$. We prove that $c>0$ and there is $U \in \mathscr{M}$ such that $\Phi(U)=c$. By $\left(S_{4}\right)$, Fatou's lemma, and the boundedness of $\left\{U_{n}\right\}$, we have

$$
\begin{aligned}
c+o(1) & =\Phi\left(U_{n}\right)-\frac{1}{2} \Phi^{\prime}\left(U_{n}\right) U_{n} \\
& =\int_{\Omega}\left(\frac{1}{2}\left(\nabla F\left(x, U_{n}\right), U_{n}\right)-F\left(x, U_{n}\right)\right) d x \\
& \geq \int_{\Omega}\left(\frac{1}{2}(\nabla F(x, U), U)-F(x, U)\right) d x+o(1) \\
& =\Phi(U)-\frac{1}{2} \Phi^{\prime}(U) U+o(1) \\
& =\Phi(U)+o(1) \geq c+o(1),
\end{aligned}
$$


where the last inequality follows from the definition of $c:=$ $\inf \{\Phi(U): U \in \mathscr{M}\}$. Hence $\Phi(U)=c$ and $c>0$ because $U \neq 0$.

Proof of Theorem 2. The functional $\Psi$ is of class $C^{1}$ on $S^{+}$by Lemma 13. It is obviously even since $F(x, U)$ is even. $\Psi^{\prime}(V)=$ 0 implies that $\widetilde{m}(V)$ is a critical point of $\Phi$. We will show that $\Psi$ satisfies the Palais-Smale condition. Suppose that $\Psi\left(V_{n}\right)$ is bounded and $\Psi^{\prime}\left(V_{n}\right) \rightarrow 0$. Then $\Phi\left(\widetilde{m}\left(V_{n}\right)\right)$ is bounded by Lemma 13(b); hence $\left\{\widetilde{m}\left(V_{n}\right)\right\}$ is also bounded by Lemma 10 . Therefore, we can take a subsequence still denoted by $\left\{\widetilde{m}\left(V_{n}\right)\right\}$ such that

$$
\begin{array}{r}
\widetilde{m}\left(V_{n}\right) \rightarrow U \text { in } W, \quad \widetilde{m}\left(V_{n}\right) \longrightarrow U \text { in } L^{q}(\Omega) \times L^{q}(\Omega), \\
1 \leq q<2^{*} .
\end{array}
$$

Employing Lemma 13 again, we have

$$
\begin{array}{r}
\Phi^{\prime}\left(\widetilde{m}\left(V_{n}\right)\right) \\
=\left\langle\widetilde{m}\left(V_{n}\right)^{+}-\widetilde{m}\left(V_{n}\right)^{-}, \cdot\right\rangle-\int_{\Omega}\left(\nabla F\left(x, \widetilde{m}\left(V_{n}\right)\right), \cdot\right) d x \rightarrow 0 \\
\text { as } n \longrightarrow \infty .
\end{array}
$$

By (56) and (57), we can easily get that $\widetilde{m}\left(V_{n}\right) \rightarrow U$ in $W$. Hence $V_{n} \rightarrow \breve{m}(U)=\left(U^{+} /\left\|U^{+}\right\|\right)$(see (48) for the definition of $\breve{m}$ ).

Let

$$
c_{k}:=\inf _{\gamma(D) \geq k} \sup _{V \in D} \Psi(V),
$$

where $\gamma$ denotes the usual Krasnoselskii genus (see, e.g., $[20,21])$ and the infimum is taken over all closed subsets $D \subset S^{+}$with $D=-D$. Since $\inf _{S^{+}} \Psi>0, c_{k}$ are well defined and positive for all $k \geq 1$. Now standard arguments using the deformation lemma, see, for example, [19-21], imply that all $c_{k}$ are critical values of $\Psi$ and $c_{k} \rightarrow \infty$ (that $c_{k} \rightarrow \infty$ is seen as [20, Proposition 9.33]). Hence, setting $U_{k}:=\widetilde{m}\left(V_{k}\right)$, we have

$$
\begin{aligned}
c_{k} & =\Psi\left(V_{k}\right)=\Phi\left(U_{k}\right)=\Phi\left(U_{k}\right)-\frac{1}{2} \Phi^{\prime}\left(U_{k}\right) U_{k} \\
& =\int_{\Omega}\left(\frac{1}{2}\left(\nabla F\left(x, U_{k}\right), U_{k}\right)-F\left(x, U_{k}\right)\right) d x .
\end{aligned}
$$

By $\left(S_{4}\right)$, the integrand above is nonnegative, so $c_{k} \rightarrow \infty$ implies that $\left|U_{k}\right|_{\infty} \rightarrow \infty$. The proof finished.

\section{Conflict of Interests}

The authors declare that there is no conflict of interests regarding the publication of this paper.

\section{Acknowledgments}

Research is supported by the Tianyuan Fund for Mathematics of NSFC (Grant no. 11326113) and the Key Project of Natural Science Foundation of Educational Committee of Henan Province under Grant 13A110015 of China.

\section{References}

[1] G. H. Fei, "Multiple solutions of some nonlinear strongly resonant elliptic equations without the (PS) condition," Journal of Mathematical Analysis and Applications, vol. 193, no. 2, pp. 659-670, 1995.

[2] A. Szulkin and T. Weth, "Ground state solutions for some indefinite variational problems," Journal of Functional Analysis, vol. 257, no. 12, pp. 3802-3822, 2009.

[3] S. Li and W. Zou, "The computations of the critical groups with an application to elliptic resonant problems at a higher eigenvalue," Journal of Mathematical Analysis and Applications, vol. 235, no. 1, pp. 237-259, 1999.

[4] D. G. Costa and C. A. Magalhaes, "A variational approach to subquadratic perturbations of elliptic systems," Journal of Differential Equations, vol. 111, no. 1, pp. 103-122, 1994.

[5] S. Ma, "Infinitely many solutions for cooperative elliptic systems with odd nonlinearity," Nonlinear Analysis: Theory, Methods and Applications, vol. 71, no. 5-6, pp. 1445-1461, 2009.

[6] S. Ma, "Nontrivial solutions for resonant cooperative elliptic systems via computations of the critical groups," Nonlinear Analysis: Theory, Methods and Applications, vol. 73, no. 12, pp. 3856-3872, 2010.

[7] A. Pomponio, "Asymptotically linear cooperative elliptic system: existence and multiplicity," Nonlinear Analysis: Theory, Methods and Applications, vol. 52, no. 3, pp. 989-1003, 2002.

[8] W. Zou, "Solutions for resonant elliptic systems with nonodd or odd nonlinearities," Journal of Mathematical Analysis and Applications, vol. 223, no. 2, pp. 397-417, 1998.

[9] W. Zou, S. Li, and J. Q. Liu, "Nontrivial solutions for resonant cooperative elliptic systems via computations of critical groups," Nonlinear Analysis, Theory, Methods and Applications, vol. 38, no. 2, pp. 229-247, 1999.

[10] W. Zou, "Multiple solutions for asymptotically linear elliptic systems," Journal of Mathematical Analysis and Applications, vol. 255, no. 1, pp. 213-229, 2001.

[11] G. Chen, "Ground state solutions of non-resonant cooperative elliptic systems with superlinear terms," Bulletin of the Korean Mathematical Society, vol. 51, no. 3, pp. 789-801, 2014.

[12] G. Chen and S. Ma, "Asymptotically or super linear cooperative elliptic systems in the whole space," Science China Mathematics, vol. 56, no. 6, pp. 1181-1194, 2013.

[13] G. Chen and S. Ma, "Infinitely many solutions for resonant cooperative elliptic systems with sublinear or superlinear terms," Calculus of Variations and Partial Differential Equations, vol. 49, pp. 271-286, 2014.

[14] G. Chen and S. Ma, "Erratum to: infinitely many solutions for resonant cooperative elliptic systems with sublinear or superlinear terms," Calculus of Variations and Partial Differential Equations, vol. 49, no. 1-2, pp. 287-290, 2014.

[15] D. G. Costa and C. A. Magalhães, "A unified approach to a class of strongly indefinite functionals," Journal of Differential Equations, vol. 125, no. 2, pp. 521-547, 1996.

[16] M. Lazzo, "Nonlinear differential problems and morse theory," Nonlinear Analysis: Theory, Methods and Applications, vol. 30, no. 1, pp. 169-176, 1997.

[17] S. Li and J. Q. Liu, "Computations of critical groups at degenerate critical point and applications to nonlinear differential equations with resonance," Houston Journal of Mathematics, vol. 25, no. 3, pp. 563-582, 1999. 
[18] C.-L. Tang and Q.-J. Gao, "Elliptic resonant problems at higher eigenvalues with an unbounded nonlinear term," Journal of Differential Equations, vol. 146, no. 1, pp. 56-66, 1998.

[19] M. Willem, Minimax Theorems, Birkhäuser, Boston, Mass, USA, 1996.

[20] P. H. Rabinowitz, Minimax Methods in Critical Point Theory with Applications to Differential Equations, vol. 65 of Conference Board of the Mathematical Sciences, The American Mathematical Society, Providence, RI, USA, 1986.

[21] M. Struwe, Variational Methods, Springer, Berlin, Germany, 2nd edition, 1996. 


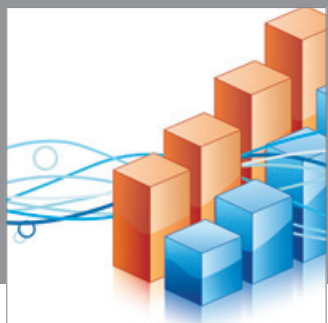

Advances in

Operations Research

mansans

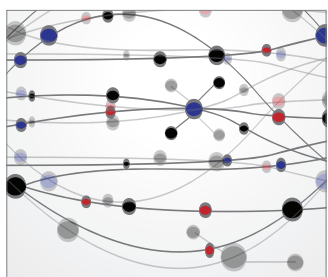

The Scientific World Journal
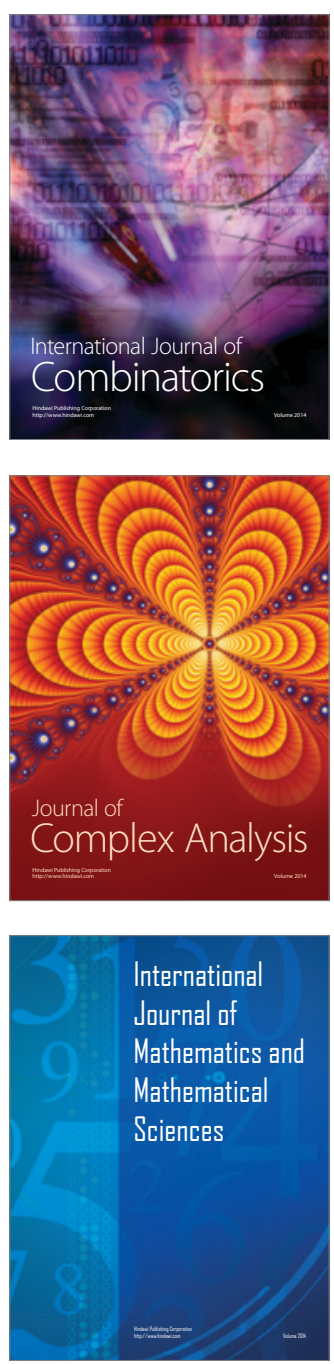
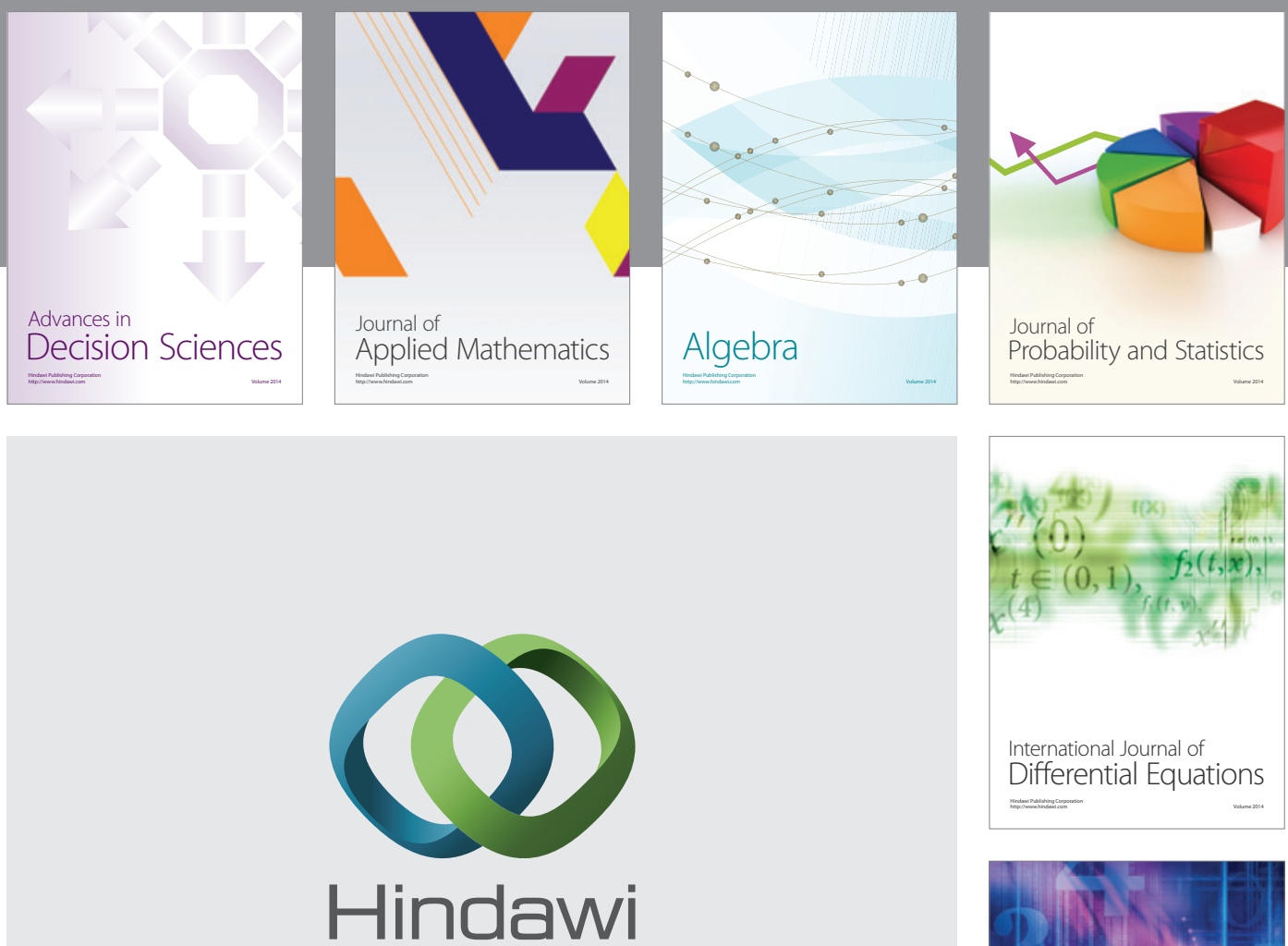

Submit your manuscripts at http://www.hindawi.com
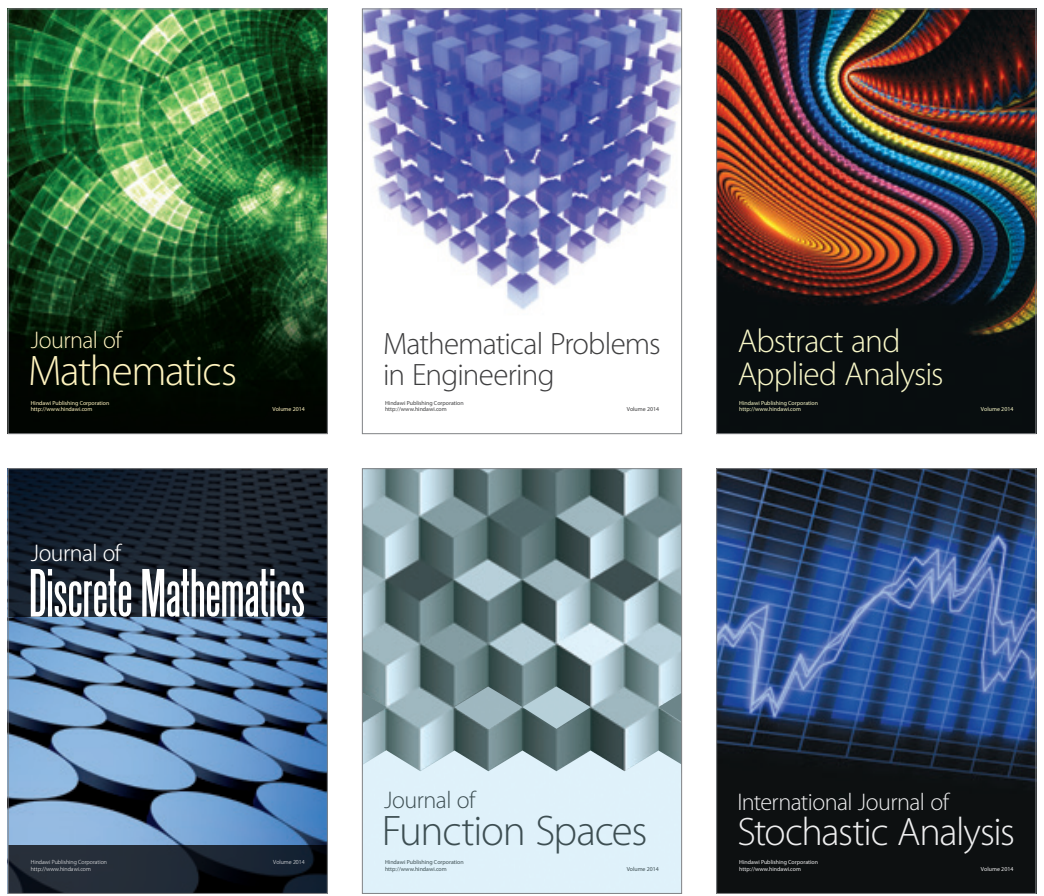

Journal of

Function Spaces

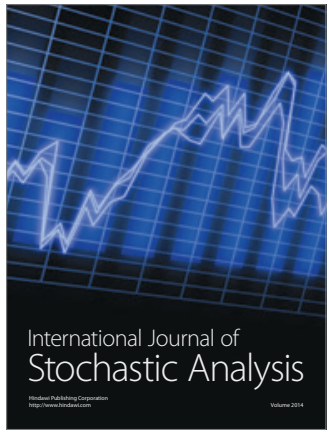

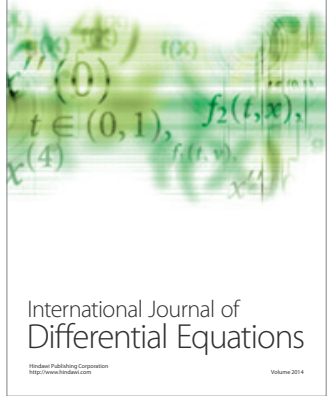
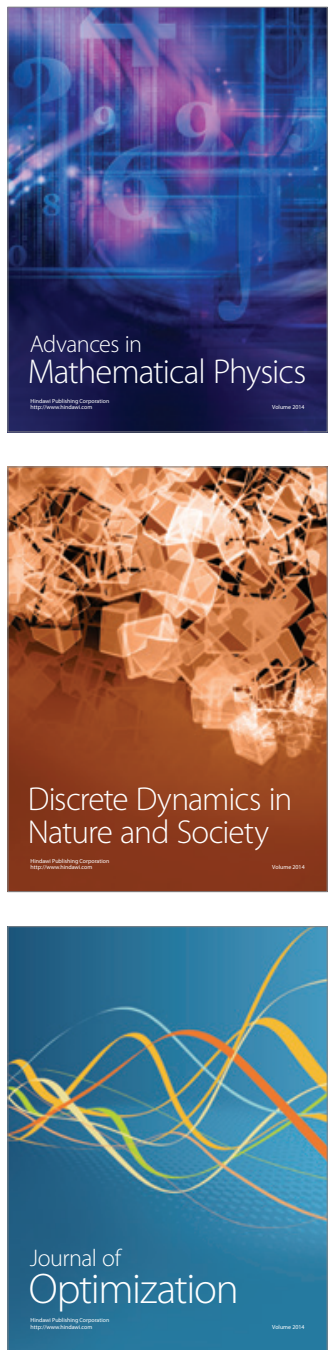